\title{
DESIGN OF A GAS SCATTERING ENERGY ANALYSER FOR THE ISIS RFQ ACCELERATOR TEST STAND
}

\author{
J P Duke, D J S Findlay, G R Murdoch, Rutherford Appleton Laboratory, England
}

\begin{abstract}
The design of an apparatus to measure the beam energy of an RFQ, based on multiple scattering of $\mathrm{H}^{-}$ions in xenon gas, is described. The purpose of the apparatus is to confirm the mean energy and energy spread of the nominal $665 \mathrm{keV}$ beam from the ISIS RFQ. This RFQ, after comprehensive testing, is intended to replace the existing Cockcroft-Walton pre-injector on the ISIS spallation neutron source at the Rutherford Appleton Laboratory (RAL). The basis of the apparatus is a set of two cascaded assemblies each consisting of a gas scattering cell, a drift length and three small apertures which together reduce the peak intensity of the beam current sufficiently to allow a semiconductor charged particle detector to be used to detect individual $\mathrm{H}^{-}$ions and measure their energies.
\end{abstract}

ISIS. One of the most important measurements to be made is of the energy distribution of the accelerated beam, and this can be done in a number of ways. The most obvious way is to use a magnetic spectrometer, and such a spectrometer is indeed being incorporated in the test stand. However, a complementary measurement method is also being sought, and this paper describes a method based on the attenuation of the particle beam with minimum energy loss and the use of a semiconductor particle detector.

The basis of the method is to use multiple scattering in a very thin scatterer of high atomic number to spread the beam out in a cone and a small aperture to select a small fraction of the cone. Two such stages are used in the present method, and this reduces the peak rate of particles sufficiently to allow a charged particle detector to be used.

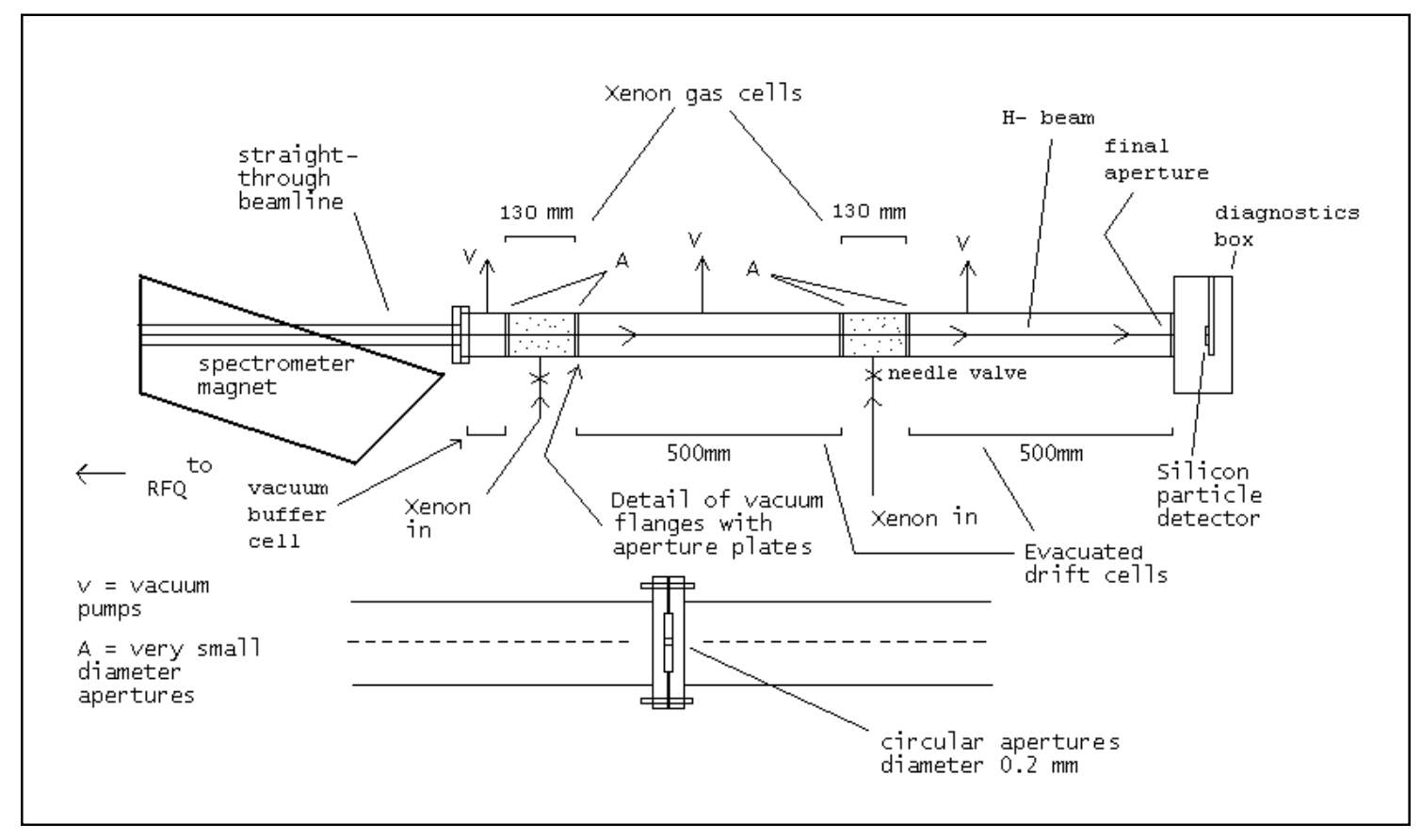

Figure 1: Schematic drawing of the whole Gas Scattering System

\section{CONCEPT}

An RFQ accelerator is to be substituted for the existing $665 \mathrm{kV}$ Cockcroft-Walton pre-injector on the ISIS spallation neutron source at the Rutherford Appleton Laboratory in Oxfordshire, England. At present the RFQ is being run on a test stand to measure its performance and demonstrate its reliability before being installed on

\section{DESIGN PROCESS}

The energies of nuclear particles may be readily measured using silicon particle detectors, but these only work well with relatively small particle fluxes such that the probability of two particles arriving within the resolution time of the detector is low. The number of $\mathrm{H}^{-}$ particles per second from the RFQ for the anticipated 
beam currents of $30-50 \mathrm{~mA}$ is $2-3 \times 10^{17} \mathrm{~s}^{-1}$ during the beam pulse. In order to satisfy the above limitation on particle rate for an expected time resolution function typically a few microseconds wide, it is necessary to reduce the rate to $\sim 1000$ per second. In other words, the original beam current must be attenuated to $3-5 \times 10^{-15}$ of its original strength. These are very small numbers, but they should be achievable using the proposed system of scattering and collimation.

As shown in Fig. 1, the gas scattering system will be placed on the straight through (zero field) beam line on the spectrometer magnet, and thus will be several metres downstream of the exit of the RFQ. Because the accelerated beam from the RFQ blows up rapidly, the flux of particles in the beam will be much reduced even with no gas in the gas scattering system. This reduction was estimated by simulating the effect of the drift in PARMILA [1] using the calculated beam at the output from the RFQ [2]. After a $5.99 \mathrm{~m}$ drift, and for currents of 30 and $50 \mathrm{~mA}$, only 210 and 181 particles respectively remained after a $1 \mathrm{~cm}^{2}$ aperture from the original 96535 or 95351 particles. On these bases, the fractions of particles from the RFQ entering the detector chamber through its $0.2 \mathrm{~mm}$ diameter aperture $5.99 \mathrm{~m}$ downstream of the end of the RFQ are $6.83 \times 10^{-7}$ for $30 \mathrm{~mA}$ and $5.96 \times 10^{-7}$ for $50 \mathrm{~mA}$. The gas scattering system is therefore required to give an additional attenuation of $7.8-5.4 \times 10^{-9}$.

The distribution of angles from a multiple scattering foil is essentially proportional to $\exp \left(-\theta^{2} / \theta_{0}^{2}\right)$ where $\theta_{0}$ is the RMS multiple scattering angle for the foil [3], and so the fraction of incident particles passing through an aperture downstream from the scatterer is a function of both the distance of the aperture from the foil and the offaxis angle. To try to minimise the likelihood of unforeseen background effects, and because for $\theta>\theta_{0}$ the multiple scattering angular dependence deviates from the simple exponential dependence given above, it was decided to avoid relying on angles far off-axis to produce large attenuation factors in a single multiple scattering stage and instead use two stages with apertures on-axis. (In principle it may be possible to use the gas scattering system to measure multiple scattering distributions.)

\subsection{Details of Calculations}

Since the present purpose is to measure the energy of the beam, it is necessary to keep the energy losses of the particles to a minimum as they pass through the scatterers so that uncertainties in corrections for energy losses do not dominate the results. Since the energy resolution of the silicon detector to be used has been measured (for alpha-particles) at $15 \mathrm{keV}$ [4], a sensible design criterion is to keep the mean energy loss $\Delta \mathrm{T}$ through the system to $\leq 15 \mathrm{keV}$, which is $2.25 \%$ of the RFQ exit beam energy of $665 \mathrm{keV}$.

In order to meet this criterion, very thin scatterers are required, and gas scatterers were thought to be most appropriate. Since the stopping power (at energies less than about $1 \mathrm{MeV}$ ) of a material of atomic number $\mathrm{Z}$ decreases as $\mathrm{Z}$ increases, whereas the RMS multiple scattering varies approximately as $\sqrt{Z}$, a gas of high atomic number should be chosen. The only practical choice is xenon, for which a $15 \mathrm{keV}$ mean energy loss would correspond to a maximum total thickness of $141.6 \mu \mathrm{g} / \mathrm{cm}^{2}$ or $0.26 \mathrm{~m}$ of gas at a pressure of 0.925 millibars. Each of the two scatterers is a gas cell in the form of a short length of beam pipe closed off at each end by a diaphragm with a small aperture at its centre.

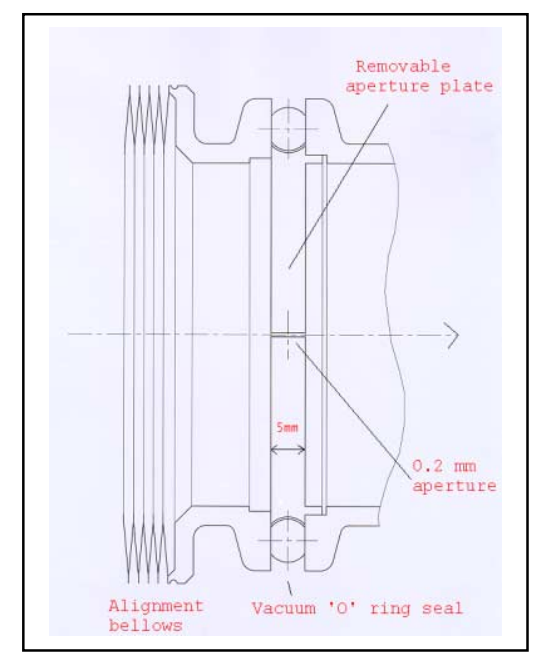

Figure 2: Detail of an aperture plate and bellows

A FORTRAN programme GASCAT2X was written to evaluate the multiple scattering distribution [3] from each xenon gas scatterer and then to calculate its integral over the corresponding exit aperture. The gas length and pressure, drift length, and aperture size were all altered until the desired attenuation was achieved.

The parameters that have been arrived at present are gas cell lengths of $130 \mathrm{~mm}$, drift lengths of $500 \mathrm{~mm}$, and xenon pressures of 0.030 and 0.033 millibars for 30 and $50 \mathrm{~mA}$ respectively. These parameters correspond to a mean energy loss of 3.25 and $3.59 \%$ of the $15 \mathrm{keV}$ limit specified above. This means that energy uncertainties should be dominated by the resolution of the detector and not by the uncertainties in calculating the gas scattering energy losses.

In order to be able to take into account the energy carried away by lost electrons, the fraction of hydrogen ions which are stripped in the xenon at these pressures was calculated, by extrapolating experimental cross section data at $0.3 \mathrm{MeV}$ [4] up to $0.7 \mathrm{MeV}$. The species fractions at the end of the gas were: $\mathrm{F}_{\mathrm{H}-}=2.5 \times 10^{-5}$, $\mathrm{F}_{\mathrm{H} 0}=9.0 \times 10^{-3}$ and $\mathrm{F}_{\mathrm{H}+}=0.991$.

The pressure in the drift spaces was chosen to be between 0.1 and $1 \%$ of the pressure in the gas cell, which implies good vacuum pumping in the drift spaces. Further, it is important to minimise leakage of xenon into the spectrometer beam line and the RFQ. Therefore a vacuum buffer cell with the same sized apertures as the gas cells has been placed upstream of the first gas cell. 
After the second gas cell, the RMS angular divergence of the beam is 6 milliradians, and the corresponding beam spot diameter after a drift of $500 \mathrm{~mm}$ is $6 \mathrm{~mm}$. Provision is being made for the final aperture and the particle detector to be moved in steps of $\sim 0.3 \mathrm{~mm}$ across the beam so that if a suitable opportunity arises then measurements can be made of the multiple scattering distribution

The maximum power deposited in the first aperture plate by the remaining beam is only $\sim 50 \mathrm{~W}$, and relatively simple cooling arrangements suffice.

The particle detector [4] will be calibrated using a $2 \mathrm{kBq}{ }^{137} \mathrm{Cs}$ conversion electron source emitting lines at 624 and $656 \mathrm{keV}$.

\section{CONCLUSION}

With the apparatus as specified above it is planned to measure the mean energy and energy spread of the nominal $665 \mathrm{keV}$ beam from the ISIS RFQ. Uncertainties should be largely dominated by the $15 \mathrm{keV}$ resolution of the particle detector. It may also be possible to measure multiple scattering distributions.

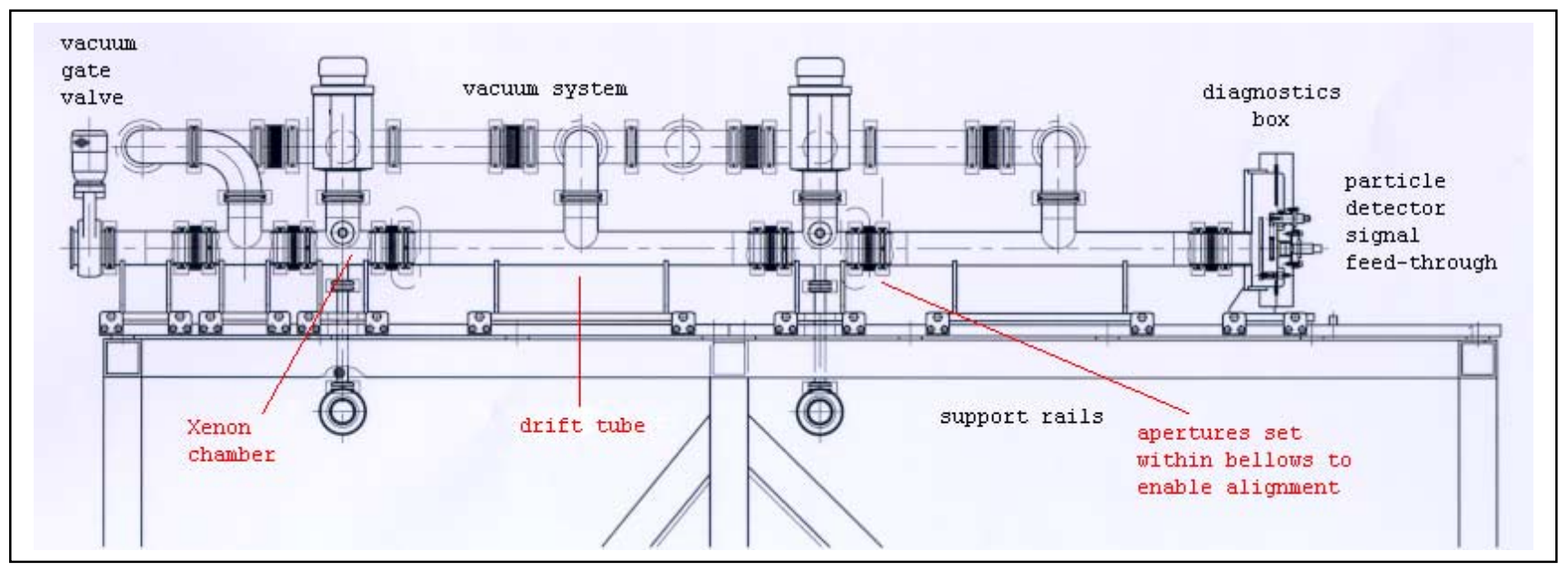

Figure 3: Engineering drawing of whole Gas Scattering System

\section{OPERATION}

It is planned to operate the system by first pumping down the whole gas scattering system beam line to low pressure, and then slowly bleeding xenon gas into the two gas cells using two needle valves. The pressure in the gas cells will be closely monitored and eventually an equilibrium will be established between the gas being bled in and the gas leaking out of the cells. The gas pressure will be adjusted to optimise the count rate from the particle detector.

\section{REFERENCES}

[1] PARMILA Linac Design Code written by Harunori Takeda, LANL.

[2] A P Letchford, private communication

[3] High Energy Particles, B Rossi, Prentice-Hall, New York 1952

[4] "An experimental study of single electron cross sections for hydrogen atoms and negative hydrogen ions in various media" - I.S.Dmitriev, Ya.A.Teplova and Yu.A.Fainberg, Journal of Experimental and Theoretical Physics, p28-29,Vol. 80, No. 1, Jan 1995.

[5] EG\&G ORTEC silicon surface barrier detectors, model no. BA-016-025-1500 